\title{
Modulated Surface Textures for Enhanced Scattering in Thin-Film Silicon Solar Cells
}

\author{
O. Isabella ${ }^{1}$, C. Battaglia' ${ }^{2}$ C. Ballif ${ }^{2}$, and M. Zeman ${ }^{1}$ \\ ${ }^{1}$ Photovoltaic Materials and Devices/Dimes, Delft University of Technology, Feldmannweg 17, 2628 CT Delft \\ ${ }^{2}$ Ecole Polytechnique Fédérale de Lausanne (EPFL), Institute of Microengineering (IMT), \\ Photovoltaics and Thin Film Electronics Laboratory, Rue A.-L. Breguet 2, 2000 Neuchâtel, Switzerland \\ e-mail: olindo.isabella@gmail.com
}

\begin{abstract}
Nano-scale randomly textured front transparent oxides are superposed on micro-scale etched glass substrates to form modulated surface textures. The resulting enhanced light scattering is implemented in single and double junction thin-film silicon solar cells. OCIS codes: $290.5825,240.6700,310.6845$,
\end{abstract}

Light scattering at textured interfaces leads to an increased optical path length of light in thin absorber layers resulting in an enhanced light absorption. Especially multi-junction thin-film silicon solar cells need an efficient scattering at long wavelengths to deliver a high photocurrent. Interface textures can be introduced into thin-film silicon solar cells by texturing glass and/or transparent conductive oxide (TCO) surfaces. Combining texturing at both glass and TCO leads to advanced surface textures. Recently, a couple of techniques have been introduced to render textured glassy substrates, such as wet [1-3] or dry etching [4] laser scribing [5] and molding of nanotextures on glass coated with transparent photo-resists [6]. We introduced a generalized approach, called modulated surface texturing, which combines different techniques, materials, and shapes in order to create the advanced surface texture [7]. This concept allows us to analyze the advanced textures, understand underlying physics for obtaining high scattering levels, and design substrates with novel surface textures.

A substrate with modulated surface texture (MST) can be prepared as a stack of layers in which a different texture is introduced at each individual interface. The first texture that has the largest features is called the background texture. The stack may comprise layers of the same or different materials and the resulting surface accommodates all lateral and vertical components. Different scattering mechanisms are superposed in a MST, resulting in higher scattering levels in a broad wavelength range in comparison to the scattering contributions from individual morphologies. To model scattering behavior of MST structures we propose a combined approach using the scalar scattering theory, that describes a wavelength-dependent exponential decay of the haze of transmitted light $\left(H_{T}\right)$, and the Mie solution of Maxwell equations, that describes scattering from objects with micrometer-scale features. The long wavelength haze value (offset effect) is determined by the background texture that can be manipulated by changing the correlation length and the peak-to-peak height of surface features.

We have fabricated a series of five MST substrates by combining large surface features of etched glass (EG, background texture), that we call EG1-EG5, with randomly-textured sputtered ZnO:Al (AZO rough, AZOR) exhibiting crater-like features (modulator). To control the surface morphology of the background textures, a sacrificial layer was applied to the glass prior to wet etching, based on a mix of $\mathrm{HF}$ and $\mathrm{H}_{2} \mathrm{O}_{2}$. With increasing thickness of the sacrificial layer textures with larger features were obtained on the glass surface. The morphological analysis of MST was carried out using the surface spatial frequency representation which took both vertical and horizontal characters of the surface features into account. Fig. 1 demonstrates that using the sacrificial layer the offset effect of $H_{T}$ can be easily controlled.

These MST substrates were tested in single and double junction thin-film silicon solar cells. The best performance of a single junction solar cell was measured for EG5 / AZOR substrate. In Fig. 2 the external quantum efficiency (EQE) of the solar cells on EG5 / AZOR and flat glass / AZOR (reference) substrates is reported. An enhanced anti-reflective effect in the short wavelength range and additional scattering in the long wavelength range due to the superposition of large and small features, are clearly observed for the cell on the MST substrate. The EG5 substrate coated with LP-CVD ZnO:B (BZO) was applied in tandem micromorph cells. In Fig. 3 a cross-sectional SEM image of the tandem micromorph cell on EG5 / BZO MST is presented. Fig. 4 shows the EQE of the tandem micromorph cell on EG5 / BZO and flat glass / BZO (reference) substrates. The EQE of the bottom cell on the EG5 / BZO MST substrate demonstrated a broadband increase in the (red) spectral response with respect to the reference flat cell.

MST substrates, also called multi-scale substrates, have been recently successfully applied in lab-scale record tandem micromorph cells [8-9] and tested in an industrial production line [10]. These results underline the potential of the MST approach for further improvement of thin-film silicon solar cells. 


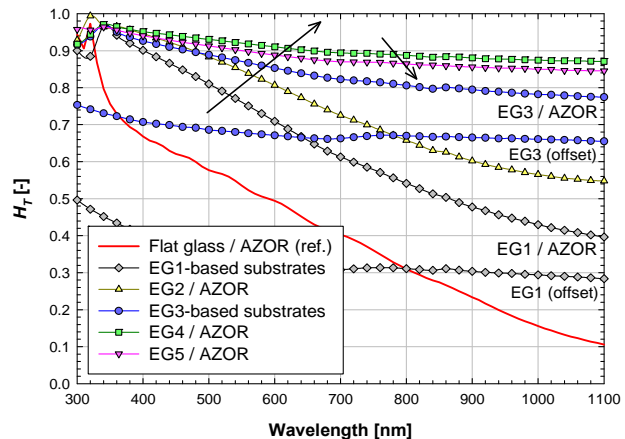

Fig. $1 H_{T}$ of the modulated surface textured substrates compared to a reference textured TCO.

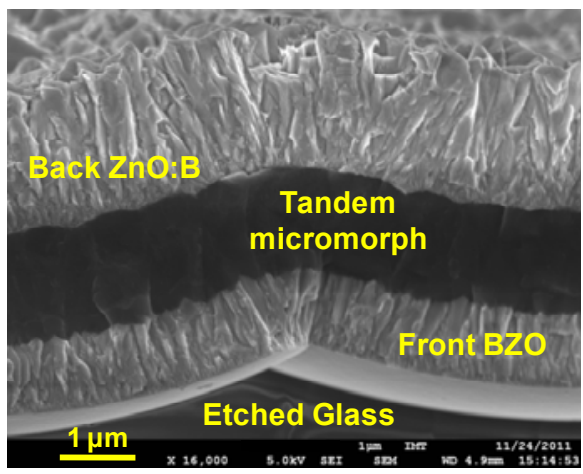

Fig. 3 Cross-sectional SEM image of the tandem micromorph cell on EG5 / BZO MST substrate.

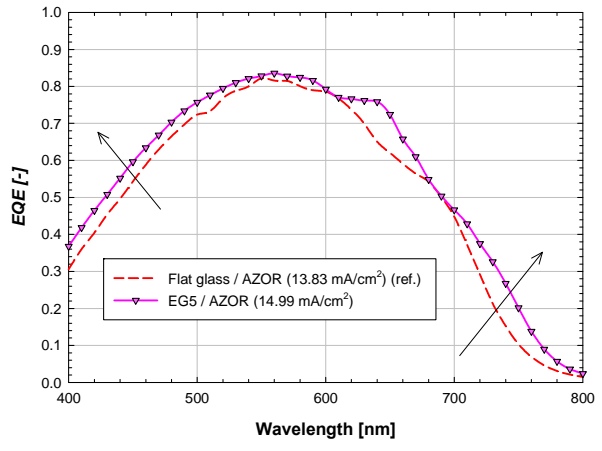

Fig. 2 EQE of single junction solar cells on reference flat glass / etched $\mathrm{ZnO}: \mathrm{Al}$ (dashed line) and on MST (EG5 / AZOR) (symbols) substrates.

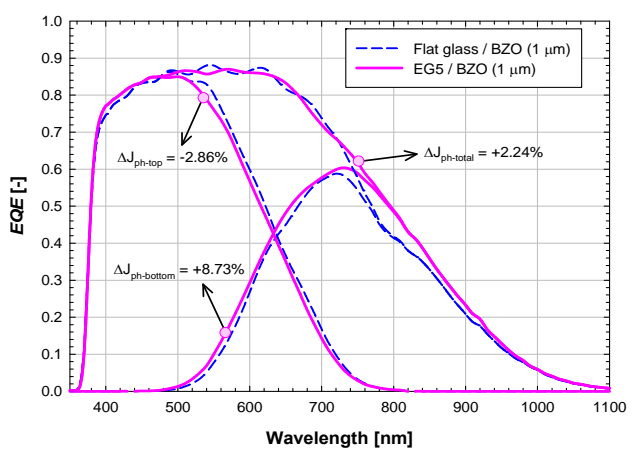

Fig. 4 EQE of tandem micromorph solar cells on reference flat glass / $\mathrm{ZnO}: \mathrm{B}$ (dashed line) and on MST (EG5 / ZnO:B) (solid line) substrates.

\section{References}

[1] O. Isabella, P. Liu, B. Bolman, et al., "Modulated surface-textured substrates with high haze: From concept to application in thin-film silicon solar cells,” 37th IEEE-PVSC, DOI: 10.1109/PVSC.2011.6186029 (2011).

[2] P. I. Widenborg and Armin G. Aberle, "Polycrystalline Silicon Thin-Film Solar Cells on AIT-Textured Glass Superstrates," Advances in OptoElectronics, vol. 2007, Article ID 24584, 7 pages, DOI:10.1155/2007/24584 (2007).

[3] Saint-Gobain Glass France, Patent WO2009/019401A2.

[4] A. Hongsingthong, T. Krajangsang, I. A. Yunaz, et al., "ZnO Films with Very High Haze Value for Use as Front Transparent Conductive Oxide Films in Thin-Film Silicon Solar Cells,” Appl. Phys. Express 3, 51102, DOI: 10.1143/APEX.3.051102 (2010).

[5] R. van Erven, R.H. Franken, J. de Ruijter, et al., "Controlled Texturing of Thin Film Silicon Solar Cell Substrates," 23rd European Photovoltaic Solar Energy Conference and Exhibition, Valencia, Spain, DOI: 10.4229/23rdEUPVSEC2008-3CO.9.4 (2008).

[6] C. Battaglia, J. Escarré, K. Söderström, et al., "Nanomoulding of transparent zinc oxide electrodes for efficient light trapping in solar cells," Nature Photonics 5, 535-538, DOI:10.1038/nphoton.2011.198 (2011).

[7] O. Isabella, J. Krč, and M. Zeman, "Modulated surface textures for enhanced light trapping in thin-film silicon solar cells," Applied Physics Letters 97, 101106, DOI: 10.1063/1.3488023 (2010).

[8] M. Boccard, C. Battaglia, S. Hänni, et al., "Multiscale Transparent Electrode Architecture for Efficient Light Management and Carrier Collection in Solar Cells", Nano Letters 12 (3), 1344-1348, DOI: 10.1021/nl203909u (2012).

[9] J. Bailat, L. Fesquet, J.-B Orhan, et al., "Recent Developments of High-Efficiency Micromorph® Tandem Solar Cells in KAI-M PECVD Reactors," 25th European Photovoltaic Solar Energy Conference and Exhibition, Valencia, Spain, DOI: 10.4229/25thEUPVSEC2010-3BO.11.5.

[10] U. Kroll, J. Meier, L. Fesquet, et al., "Recent Developments of High-Efficiency Micromorph Tandem Solar Cells in KAI-M/ Plasmabox PECVD Reactors," 26th European Photovoltaic Solar Energy Conference and Exhibition, Hamburg, Germany, DOI: 10.4229/26thEUPVSEC2011-3BO.2.6 (2011). 